\title{
Analysis of technogenic risks hazard production facilities using soft computing
}

\author{
Oleg Nikolsky ${ }^{1}$, Nikolay Vorobyev $^{1, *}$, Lydia Kulikova ${ }^{1}$, Stanislav Khomutov ${ }^{1}$ \\ ${ }^{1}$ Polzunov Altai State Technical University, pr-t Lenina, 46, Barnaul, 656038, Russia
}

\begin{abstract}
A new concept has been found for the evaluation and management of risk hazard of electrical installations for a real production object on the basis of using soft computing. This approach refers to the use of managing tasks, the basis of which lies in the theory of taking decisions in indefinite conditions.
\end{abstract}

\section{Introduction}

Provided that safety is key in various areas of work. The analysis of reasons for accidents and injuries in spite of their seemingly occasional occurrence complies with concrete principles $[1,2]$. As a rule, electrical installation accidents are preceded by a period of the accumulation of defects caused by the aging and wearing out of equipment, the influence of environmental negative factors, mistakes by electro technological staff (here will be reviewed the hazardous technogenic situations caused by the electricity).

It is known that the classical theory of reliability takes into account element content of the technical system, functional links, schedule of work, etc. However, this theory leaves apart such important components of a complex system as the environment and human factor which are dominating in occurring hazardous situations (fire, electrical damage). Today it is known [3] that more than $70 \%$ of all accidents are connected to the mistakes and erroneous actions of support staff. In addition, the lack of effective control methods of technical condition of electrical installations leads to the fact that staff have to take intuitive decisions to prevent hazardous situations. The problem is created on the one hand by the objective difficulty of the identification of the hazard of man-machine system functioning in uncertain conditions [4] and on the other hand by the lack of a concept for evaluating and predicting risks.

For the quantitative evaluation of electrical installations hazard it is proposed [5] to use a logical-linguistic approach, the basis of which has a symbolic language of logics and formalism of probability analysis. And the hazard of electrical installations is offered to evaluate with the help of the quantitative indicator of risk $R_{\Sigma}$, which presents a two-

${ }^{*}$ Corresponding author: vnprol51p@yandex.ru 
parameter model combining the hazardous situation $P_{i}$ and its consequence-the damage $Y_{i}[6]$, that is

$$
R_{\Sigma}=\sum_{i}^{n} P_{i} Y_{i}
$$

If there is $n$ of hazardous situations $i$ with various probabilities $P_{i}$ and the corresponding damage $Y_{i}$.

In some cases risk can be estimated in mathematical damage or loss expectancy

$$
R_{\Sigma}=\int F(Y) P(Y) d Y
$$

Where $F(Y)$ - is a weight function of losses with the help of each the consequences of hazards of different nature (including material loss because of a person's death) are equaled to one cost evaluation, expressed in monetary equivalent; $P(Y)$ is the density of distribution of occasional value $Y$.

Known methods of technogenic risks analysis do not take into consideration the uncertainty of original factors conditioned by the breakdown of the equipment, over normative environmental factors and staff mistakes which doesn't allow for the provision of control and management for the risk of accidents and electro injuries at production in the scope of real time. The difficulty of the problem of evaluation a $d$ management of technogenic risks of electrical installations is defined by the fact that the person taking the decision faces the following circumstances:

1. low structuring and physical inhomogeneity of component in the reviewed manmachine system ( in our case the system "Man-Electrical installation-Environment");

2. numerous restrictions of technological, normative and economical nature;

3. occasional initiative prerequisites leading to hazardous technogenic situations;

4. uncertainty of the basic data and lack of satisfactory descriptions of occurrence and development of hazardous technogenic situations which makes it practically impossible to take adequate risk evaluation.

Uncertainty of the information is caused by the state of environment conditioned by an occasion. Ambiguity is the consequence of process subjectivity of human thinking and semantical diversification and structure of a natural language.

A significant difficulty of modern technical systems with the participation of a personoperator dictates the refusal from building traditional probability models and attracting corresponding mathematical instrument. The use of bee informational technologies seems prospective- such as soft computing elaborated by L. Zade [7].

It is known that the basis of mathematical analysis lies in admitting the hypothesis of infinite divisibility of time and space which contradicts modern thinking [8].

The essence of the reviewed approach unlike traditional hard computing connected with solving differential and integral equations - is oriented at objects of real world, mainly macroscopic. Soft computing can be referred to the technologies when for example technogenic environment of a real object cannot be described with the concrete expressions [9].

Which of the reviewed approaches is more objective, that corresponds to the world? There is an opinion that classical models of physical processes describing for example electromagnetic field with the help of Maxwell's differential equations are quite strict and accurate; and methods of computational mathematics are considered as approximate solutions of the equations. However, practice shows that during modeling of real objects 
often there is an easier method of searching for an approximate decision; introducing corresponding faults -and the accurate decision based on use of classical mathematical analysis can be seen as some sort of idealization. In this case, it's enough to find an approximate (soft) solution and research the object with it finding accurate (strict) solution. Then there is no necessity in the last at discussing practical tasks. Such conclusion is proven by the L. Zade formulated principle of incompatibility: with the growth of system (object) complication decreases the possibility of its accurate description and its practical necessity [10].

In favor of using soft computing instrument is also the fact that original databases which are the basis for building a model always loses approximate character because they can be received either by measuring (with inevitable errors), or by experts which is subjective.

The aim of the research is the elaboration of methods and instruments of support for taking decisions for evaluation and management of technogenic risks of hazard of electrical installations of real objects in the conditions of uncertainty of the original data and possibly not stationarity of current processes. The mentioned aim is realized on the base of building a new theory of technogenic risks of system "Man-Electrical installation-Environment ".

\section{Theoretical explanation of use of soft computing procedure for evaluation and prediction of hazard risks of electrical installations}

According to [10] the procedure of soft computing presupposes the use of definite mathematical instruments, some of which one can outline uncertain sums, uncertain logics, neuron beta and evolutional computing. Soft computing should be used for approximate description of a complex object (in our case system "Man-Electrical installationEnvironment") as some compensation to the absent static base.

Let us remember the definition of uncertain sums presenting the class of objects in which there is vague boundary between the objects which are in this class, and those which are not. It is possible to formalize this statement as follows.

Let $X=\{x\}$ is a combination of objects marked through $x$. Then the uncertain $A$ in $X$ is the combination of regulated pairs of $A=\left\{x, \mu_{A}(x)\right\}, x \in X$ where $\mu_{\mathrm{A}}(x)$ is the degree of attachment of $x$ to $A$, and $\mu_{A}: X \rightarrow M$ is the function reflecting $X$ in the space $M$ named the space of attachment.

When $M$ has only 2 points 0 and 1 (boundaries of dome interval), the sum $A$ is certain and its function of attachment corresponds with the characteristic function of a usual sum.

If $M$ is an interval $(0,1)$, where 0 and 1 is the lowest and highest degree of attachment then one can consider that the uncertain sum $A$ in spite of the ambiguity of its boundaries can be clearly defined by matching each object to the number $x$, lying between 0 and 1 which presents the degree of its attachment to $A$.

So, the significance of the function $\mu(x)$ is interpreted as the degree of attachment of a point to the uncertain $\operatorname{sum} A$.

The theory of uncertain sums is referred to by the term "Fuzzy Logic" which means unclear or vague logic [7].

The main task of the uncertain logic is in the possibility of presenting a natural language (human thoughts) possessing numerous interpretation into simple logical constructions.

It enables one to hold the analysis of the system "Man-Electrical installationEnvironment " not using strict mathematical models by simplified approaches receiving the approximate solution. 
That is why unclear logic should be applied at the evaluation of technogenic hazard of electrical installations on real production objects when the role of the human factor is dominating.

In this case using logical model-phrases "If...then..", one can give semantical description of the observed system: "Man-Electrical installation-Environment ".

As an illustration of the mentioned statements in the Figure 1 there is the interpretation of evaluation of the integral risk of the system: "Man-Electrical installation-Environment ". Here the integral risk $R_{\Sigma}$

$$
R_{\Sigma}=R\left(Y_{C}\right)+R\left(Y_{M}\right)+R\left(Y_{E}\right)
$$

The indicator of potential hazard taking into account social $R\left(Y_{C}\right)$, material $R\left(Y_{M}\right)$ and ecological $R\left(Y_{E}\right)$ losses presented in the financial equivalent [11].

Let us express the integral risk $R_{\Sigma}$ as

$$
R_{\Sigma}=F[\bar{X}, \bar{Y}, \bar{Z}]
$$

Where $\bar{X}$ is the possibility of hazardous situation; $\bar{Y}$ - the duration of the effect of the hazardous factor on the object; $\bar{Z}$-consequences characterized by the measure of difficulty of the situation.

The overviewed linguistic evaluation $R_{\Sigma}$ in the form of a.3 dimensional matrix enables to consider the opportunity (soft probability) of some hazardous situation, for example a person's getting under voltage, duration of the electrical influence on the human body and consequences characterized by the measure of difficulty of situation (light electrical injury, invalidity, death).

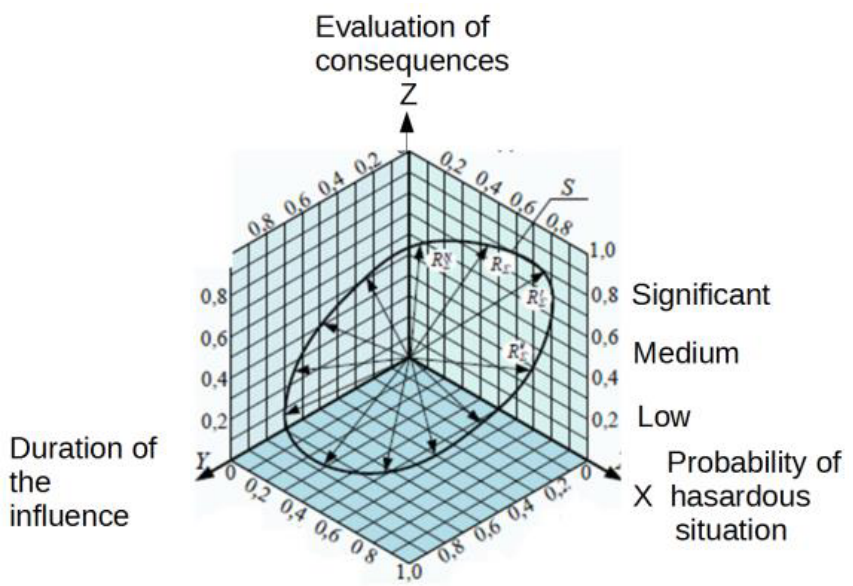

Fig. 1. Linguistic evaluation of the integral risk.

The basic principles of ontological modeling of the system: "Man-Electrical installationEnvironment".

Let us present the functional process of the overviews man-machine system in terms of limited natural language with the help of a linguistic variable meanings of which can be numbers and words (word combinations) of some natural or artificial language [12]. In it's essence s linguistic variable is a descriptive tuple

$$
L V=[L, T, X, G, M]
$$


Where $L V$ is a linguistic variable, $T$ - is a multitude of its meanings (term multitude), $X$-is the universal multitude of uncertain variables, $G$ - is the syntactic procedure of the new term formation allowing to build compound terms our of simple atom terms, $G: T \rightarrow T^{*}, T^{*}$ is the wide term multitude, $M$ - is a semantical procedure forming uncertain sums for each term $T$ of the given linguistic variable

Uncertain logical-linguistic model (5) is realized into the expert-programme complex (Figure 2) aimed automatization of technogenic risks computing during projecting the systems of safety of electrical installations or taking decisions at the exploitation of a manmachine system.

In the system of uncertain conclusion an important role is played by the procedure of phasification- changing of the initial parameters (risk forming factors) into uncertain sum [13].

The procedure of soft computing is the expert's behaviour taking decisions-that is provides the information about the technical condition of the electrical installations of a real production object,

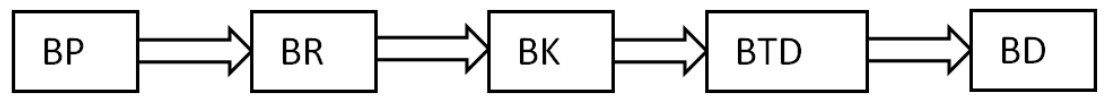

Fig. 2. The organization of the expert-programme system.

BP-block of phasification, BR-base of logical rules, BK-base of knowledge (functional attachment), BTD-block of taking decisions (block of the uncertain logic conclusion), BDblock of dephasification including interactive diagnostics of the violation causes and choice of necessary action aimed at its removal. The algorithm of defining $R_{\Sigma}$ system contains the following steps:

1. Forming the table of risk forming factors of the system components for each cluster. A linguistic variable is introduced as -"The significance of a risk factor " on the base of a term-multitude meanings such as "low"-L, medium -M, high-H.

Also, there is grouping of the received combination of risk-forming factor according to clusters, which characterize basic indicators of functioning of the overviewed man-machine system. Each cluster is formed into a corresponding vector:

- component "man"

$$
\overline{V_{C H}}=\sum_{i=1}^{n}\left(X_{i}\right), n=\overline{1-12} ;
$$

- component "electrical installation"

$$
\overline{V_{E Y}}=\sum_{j=1}^{m}\left(Y_{j}\right), m=\overline{1-8} \text {; }
$$

- component "environment"

$$
\bar{V}_{C}=\sum_{e=1}^{p}\left(Z_{e}\right), p=\overline{1-5}
$$

Where $n, m, p$ - is the amount of risk-forming factors in the components of the system:

"Man-Electrical installation-Environment".

2. Holding the expert evaluation: each risk forming factor (the number of the multitude is given a semantic expression, corresponding code and weight, hazard indicator (normative evaluation) expressed by the number lying between 0 and 1 (its sum should be equal to 10). 
3. Counting the risks of each component / cluster of the man-machine system

$$
R_{k}=\sum_{k=1}^{S} \mathrm{w}_{\mathrm{i}} r_{k}
$$

Where $\mathrm{w}_{\mathrm{i}}$ - is the indicator weight $\left(\Sigma w_{i}=1\right) ; S$ - is the amount of risk forming factors included into each cluster.

The significance of a risk $r_{k}$ is determined based on the ranking and convolution.

4. Integral risk of the taken linguistic variable "the significance of the risk factor" with the term-multitude of meanings $T_{i}$ for standard 3 level classificator with 3 functions of attachment $\mu_{\mathrm{ij}}\left(X_{i}\right)$ of s triangular view is defined by the expression

$$
R_{\Sigma}=\sum_{i=1}^{6} \mathrm{w}_{\mathrm{i}} \sum_{j=1}^{3} \beta_{j} \mu_{\mathrm{ij}}\left(X_{i}\right)
$$

Where $\mathrm{w}_{\mathrm{i}}$ weight of the $i$ factor in the pack, $\beta_{j}$ - key points of a standard 3 level classificatory, $\mu_{\mathrm{ij}}\left(X_{i}\right)$ - the meaning of functioning attachment of $j$ quality level.

On the base of the mentioned there was elaborated the system of diagnostics of the technical condition of electrical installations.

The object of research is a typical electrical establishment (electrical nets and technological electrical installation of a bird house) overviews by us as a man-machine system: "Man-Electrical installation-Environment ".

The reviewed method and support algorithm based in the use of logical- linguistic models enable to raise the reasonability of the taken decisions in conditions of uncertainty of the initial data.

\section{Practical realisation}

Below there is given the example of the use of soft computing instrument which is an adequate instrument for description of technogenic hazards of real objects. For the evaluation of integral risk $R_{\Sigma}$ let us introduce 3 point linguistic scale (Table 1) with interval meanings according to the normative- technical documentation.

Table 1. Linguistic scale of integral risk evaluation.

\begin{tabular}{|c|c|}
\hline Type of integral risk $R_{\Sigma}$ & The evaluation of risk (per year) $R_{\Sigma}=10^{-q}$ \\
\hline Low & Satisfactory $-\left[10^{-5}-10^{-6}\right]$ \\
\hline Medium & Acceptable $-\left[10^{-3}-10^{-5}\right]$ \\
\hline High & Unacceptable $-\left[10^{-2}-10^{-3}\right]$ \\
\hline
\end{tabular}

Note: $R_{\Sigma}$, the evaluation of risk, $q$ - module of frequency of events per year, in future it will be noted as module $q$ without account of sign, giving the sign after counting the module $q$ "

The concept of defining hazardous technogenic situations on the basis of uncertain logics is formed on the supposition of the system that Man, Electrical installation, Environment, legislative and normative base, Macroeconomic numbers and Innovative numbers influence the hazardous technogenic situations applying to the electrical installations of a real production object forming risk factors. 
Consequently a system was put in place for uncertain logical conclusion for evaluation of hazardous technogenic situations applied to electrical installations of real objects on the base of Fuzzy toolbox 0.4.6 of programme Scilab 5.2.2.

The hierarchical structural scheme of uncertain logical conclusion of the expert system of evaluation of hazardous technogenic situations applying to electrical installations of a real production object- is shown in Figures 3,4.

The indicator of event frequency degree per year a $q$ is formed in the system of uncertain conclusion sugeno_tip_calculator_q.

Entering vector is formed on the base of the expert evaluation of risk forming factors and directed it to the system of uncertain logical conclusion, at the end of which there is the indicator of event frequency degree $q$, and on its base the real meaning $R_{\Sigma}$ - the kind of integral risk and risk evaluation (Table 1).

Due to the lack of information about risk-forming factors for it's linguistic evaluation there were used 5 terms of triangular distribution function.

The elaborated the system of uncertain logics Risk 2_2015_07_20_final_16_c_Kn_N_Chiken enables to use certain entering numbers: $n$ - low $(0), n s-$ lower than average (2.5), $s-$ average (5), vs - higher than average (7.5), and $v-$ high (10).

The disposal of risk indicator $q$ from the range 0-10 to the range 2-6 (according to the Table 1) is done with the help of Normalizator in the format Scilab.

For getting the results of uncertain conclusion (S_1_2-> Intermediate result 1 - Figure 3 ) by the given risk forming factors there are used uncertain databases Sugeno which are the same for all systems of uncertain logics since they provide the largest linearity of firming the logical packs.

The meaning of risk-forming factors are connected with the logical function, weight coefficient of the corresponding rule is Equal to 1.

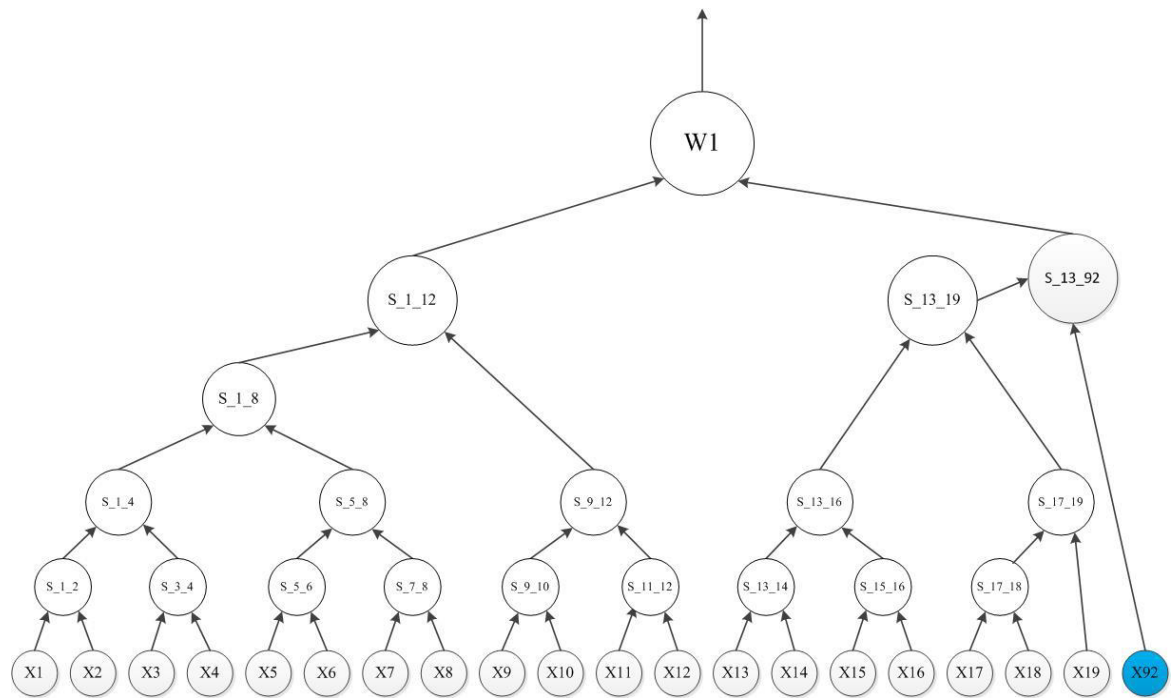

Fig. 3. Hierarchical structure scheme of uncertain logical conclusion of expert system of hazardous technogenic situations applied to electrical installations (beginning).

Where $X 1-X 92$ - risk forming factors; $S 1$ 1_12 - logical pack corresponding to $5 \mathrm{~h} 3$ risk from the component of the system: "Man"; $\bar{S} 1-13-92$ - from the component "Electrical installation"; W1- from the components of system "Man" and "Electrical installation". 


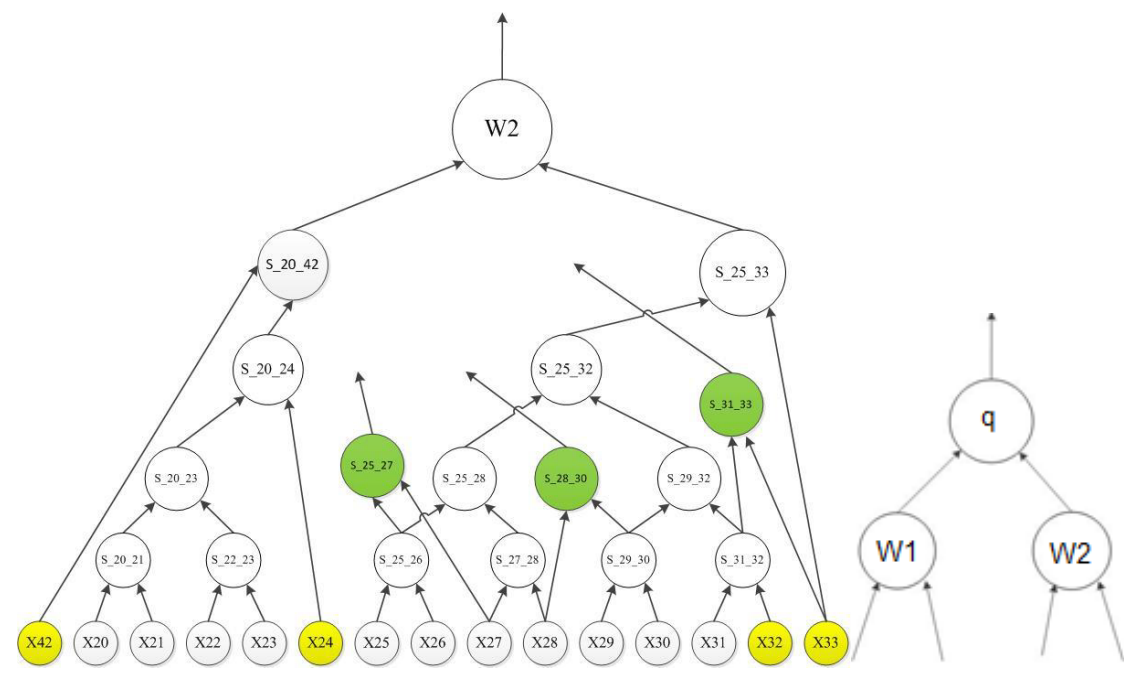

Fig. 4. Hierarchical structure scheme of uncertain logical conclusion of the expert evaluation system of hazardous technogenic situations applied to electrical installations of a real production object (the end).

Where $X 42-X 33$ - risk-forming factors; $S 1 \_20 \_42$ - logical pack corresponding to the risk of the component of the system "Environment" correspondingly, $-S 25$ - 27 - from the component "Legislative and normative base ", $S 2830$ - from the component "Macroeconomic numbers", S31_33 - from the component "Innovative numbers "; W1, W2intermediary logical packs, $q$ - risk indicator

The offered scheme of forming the database reflecting the hierarchical connection between entering variables $(X 1-X 33)$ and outcoming variable $q$ enables to evaluate the technical condition of the electrical equipment of a real object and evaluate the hazardous technogenic situation $R_{\Sigma}$. After starting the performance of the file sugeno_tip_demo1.sce as a result of uncertain logical conclusion of risk-forming factors in the common window of Scilab they get the evaluation of a hazardous technogenic situations applied to electrical equipment of a real object in the form of a $q$ - the event frequency indicator per year , and in its base $R_{\Sigma}$ - the evaluation of hazardous technogenic situations applied to electrical equipment of a real production object on the base of which the management of the company takes decisions either about the continuing the exploitation of the electrical equipment of the enterprise or (on in the base of intermediary logical packs) about the necessity of risk management at the enterprise by changing the corresponding(most problematic) technogenic processes. This, at researching electrical equipment of a birdhouse the risk indicator turned out to be $\mathrm{q}=2.84$ that is high $\left(R_{\Sigma}=10^{-2.84}\right)$ according to the Table 1 . As a result of changing the most problematic technogenic processes there was a range of riskforming factors forming logical packs $S 1_{-} 12, S 13_{-} 92$ and $R_{\Sigma}$ decreased to the number $R_{\Sigma}=10^{-3.67}$ which can be admitted as satisfactory meaning of the integral risk of electrical installations of a birdhouse.

The elaborated above programme methods realise the offered approach and support procedures for taking decisions in risk management of a production installation. 


\section{Conclusion}

The analysis and diagnostics of the technical condition of production objects is starting to become more widely used, aimed at finding the weak points for understanding the present measures in preventing accidents and injuries at production enterprises and objects of social environment. It is shown that the efficiency of the functioning of man-machine systems "Man-Electrical installation-Environment " can be expressed with the integral risk which to apply the evaluation mechanism of economic efficiency of system optimization of the safety of electrical installations.

The base for the integral risk evaluation is the multi parameter description of the system condition, considering not only reliability but also its safety, ergonomic and economic features. For the example of practical computing there was identified a realization of management functions of the integral risks of electrical installations of a real production object.

\section{References}

1. O.K. Nikolsky, Systems of providing safety of electrical installations up to $1000 \mathrm{~V}$ (Publishing house of Altai state technical University, Barnaul, 2000)

2. The basis of electromagnetic combinability: students book for high schools (Altai printing plant, Barnaul, 2007)

3. S.A. Detina, Reliability of operative staff during operative shifts (Samara, 2012)

4. O.K. Nikolsky, Vestnik of ASAU 3, 102 - 106 (2014)

5. T.V. Eremina, Probability analysis of safety of village electrical installations (Publishing house of East-Siberian state technical University, Ulan-Ude, 2010)

6. O.K. Nikolsky, Polzunovskii Vestnik 4, 100 - 106 (2014)

7. L. Zade, The term of a linguistic variable and its use at taking approximate solutions (Mir, Moscow, 1976)

8. M.M. Lavrentjev, Incorrect tasks of mathematical physics and analysis (Science, Moscow, 1980)

9. D.A. Molodtsov, Proceedings of the Academy of Sciences 6, 137 - 144 (2001)

10. L. Zade, Artificial intelligence news 2-3, 7-11 (2001)

11. O.K. Nikolsky, Theory and practice of managing technogenic risks: student book (Publishing house of Altai state technical University, Barnaul, 2015)

12. N.V. Diligentsky, Mechanical Engineering 1, 322-336 (2004)

13. R. Bellman, Taking decisions in vague conditions (Mir, Moscow, 1976) 